\title{
Fuzzy Principle Algorithm based on Mathematical Theory
}

\section{Zhang Zhiliang}

Linyi University, Yishui, Shandong, 276400

Keywords: Fuzzy reasoning algorithm; Fuzzy mathematics; Fuzzy control; Mathematical principles

\begin{abstract}
Fuzzy reasoning algorithm is the key content in fuzzy logic research, and is also the basic theory in fuzzy control. With the deepening of the research, currently a lot of fuzzy reasoning algorithms are proposed and applied in electrical appliances and many industrial productions. But its basic research and theory are relatively weak. This paper studies fuzzy reasoning algorithm based on the principle of mathematics and verifies three theorems. The results show that there is a bounded real function between the conclusion and premise of a variety of fuzzy reasoning algorithms, and different algorithms are the transformation in structure form of the function. This verifies the reliability of fuzzy reasoning algorithm.

Professor L.A.Zadeh at the University of California in 1965 first put forward the basic theory and thought of fuzzy mathematics, which laid the foundation of fuzzy mathematics. In 1973, it was first applied in control. Zaheh developed the way to apply fuzzy reasoning on the basis of fuzzy mathematics and it played an important role in analyzing and solving the problems of FMP and FMT. This method was applied in fuzzy control afterwards. The widely application of fuzzy reasoning also makes it the important position in fuzzy system theory study. Currently, the most widely used method in industrial production is still CRI method proposed by professor Zadeh, but this method has some problems which also lead people to urgently seek for better reasoning algorithms. For decades, CRI method is expanded and constantly fused into more new compound operation methods and fuzzy definition relations, and a variety of fuzzy reasoning methods are obtained. In addition to basic CRI method, there are analogical reasoning algorithm based on similarity degree, interval-valued reasoning methods, and evidence reasoning method. J.F.Baldwin highlights logic characteristics of approximate reasoning, and puts forward a method based on true value limit. There are also a lot of research at home, for example, professor Wang Guojun put forward a kind of brand-new full implication triple I algorithm in 1999 in our country on the basis of in-depth study of CRI algorithm. In addition to further correction of CRI, it also innovatively puts forward the research direction of logical semantic implication.
\end{abstract}

\section{CLASSICAL ALGORITHM STUDY OF FUZZY REASONING}

\section{A. FMP model and CRI algorithm}

FMP, namely fuzzy modus ponens, is the basic model for fuzzy reasoning algorithm and the basic format is expressed as:

Hypothesis I: if $\mathrm{x}$ is $\mathrm{A}$, then $\mathrm{y}$ is $\mathrm{B}$,

Hypothesis II: if $\mathrm{x}$ is A ', conclusion y is B', and the formal representation is as follows:

$$
A \rightarrow B, \frac{A^{*}}{B^{*}}
$$

Here $\mathrm{A}$ and $\mathrm{A} *$ are the fuzzy set of $\mathrm{X}$ discourse, and the same $\mathrm{B}$ and $\mathrm{B} *$ are the fuzzy set of $\mathrm{Y}$. Zaheh first put forward CRI method to solve the above FMP problem, and its basic idea is: 
For the known condition's transformation in FMP problem, transform $A \rightarrow B$ into an $X \cdot Y$ fuzzy relation $\mathrm{R}(\mathrm{X}, \mathrm{Y})$, namely it can be replaced by a mapping $\mathrm{R}: \mathrm{X} \times \mathrm{Y} \rightarrow[0,1]$ :

$$
R(x, y)=R(A(x), B(y))
$$

Get $\mathrm{B} *$ through fuzzy relation $\mathrm{R}$ and $\mathrm{A} *$ :

$$
B *(y)=A^{*} \circ R(A(x), B(y))
$$

This is the basic CRI algorithm model, and $\circ$ is a synthesis operation. It is also called sup-* algorithm in fuzzy control algorithm. The $*$ is a triangle norm. The most common real multiplication and $\wedge$ in current control theory are applied as a substitute. If use $\wedge$ as a substitute for *, you can get:

$$
B^{*}(y)=\sup \left[A^{*} \circ R(A(x), B(y))\right], \quad x \in X
$$

Here $\mathrm{B} *$ can be thought of as both the CRI solution of the problem, which is commonly referred to as R-mode CRI solution. For compound operation, use triangle modulus method, and implication operator can choose other operators apart from Rz.

\section{B. Multiple and multidimensional fuzzy reasoning model}

There are other fuzzy patterns except for FMP, maily McFMP and mdMFP two kinds, respectively multiple fuzzy inference and multidimensional fuzzy inference. The basic algorithm and format is:

First, the general format of mcFMP is expressed as:

Hypothesis 1: if $\mathrm{x}$ is $\mathrm{A} 1, \mathrm{y}$ is $\mathrm{B} 1$,

If $\mathrm{x}$ is $\mathrm{A} 2, \mathrm{y}$ is $\mathrm{B} 2$,

If $\mathrm{x}$ is $\mathrm{An}, \mathrm{y}$ is $\mathrm{Bn}$.

Hypothesis 2: $\mathrm{x}$ is $\mathrm{A} *$, and the conclusion $\mathrm{y}$ is $\mathrm{B}^{*}$.

Formal presentation formats is as follows:

$$
\begin{aligned}
& \mathrm{A} 1 \rightarrow \mathrm{B} 1, \\
& \mathrm{~A} 2 \rightarrow \mathrm{B} 2 \\
& \ldots \\
& \mathrm{An} \rightarrow \mathrm{Bn}, \frac{A^{*}}{B^{*}} .
\end{aligned}
$$

$A *$ and Ai are the fuzzy sets on the discourse $X, B *$ and Bi are $Y^{\prime}$ 's fuzzy sets $(i=1,2,3 \ldots n, n$ $\geq 2$ ). Then the solution based on Zaheh is: 


$$
\begin{aligned}
& B^{*}=A^{*} \circ \bigcap_{i=1}^{n}\left(A_{i} \rightarrow B_{i}\right), \\
& B^{*}(y)=\underset{x \in X}{\vee}\left[A^{*}(x) \wedge\left(\bigwedge_{i=1}^{n} R_{\mathrm{z}}\left(A_{i}(x), B_{i}(y)\right)\right)\right]
\end{aligned}
$$

The solution based on Dubois-Prade is:

$$
\begin{aligned}
& B^{*}=A^{*} \circ \bigcap_{i=1}^{n}\left(A_{i} \rightarrow B_{i}\right), \\
& B^{*}(y)=\stackrel{n}{\wedge} \underset{i=1}{\vee}\left[A_{x \in X}^{*}(x) \wedge\left(\wedge_{i=1}^{n} R_{\mathrm{DP}}\left(A_{i}(x), B_{i}(y)\right)\right)\right]
\end{aligned}
$$

The general format of mdFMP is:

Hypothesis 1: if $\mathrm{x}$ is $\mathrm{A} 1$, if $\mathrm{x}$ is $\mathrm{A} 2 \ldots$ if $\mathrm{x}$ is $\mathrm{An}, \mathrm{y}$ is $\mathrm{B}$. Hypothesis 2 : if $\mathrm{x}$ is $\mathrm{A} * 1$, if $\mathrm{x}$ is $\mathrm{A} * 2 \ldots$ If $\mathrm{x}$ is $\mathrm{A} * \mathrm{n}$, the conclusion is: $\mathrm{y}$ is $\mathrm{B} *$.

Formal expression is:

$$
\begin{aligned}
& \mathrm{A} 1, \mathrm{~A} 1, \mathrm{~A} 1, \ldots, \mathrm{An} \rightarrow \mathrm{B} \\
& \frac{A_{1}^{*}, A_{2}^{*}, \ldots, A_{n 1}^{*} \rightarrow B}{B^{*}}
\end{aligned}
$$

$A * i$ and $\mathrm{Ai}$ are the fuzzy sets on the discourse $\mathrm{X}, \mathrm{B} * \mathrm{I}$ and $\mathrm{Bi}$ are $\mathrm{Y}^{\prime}$ s fuzzy sets $(i=1,2,3 \ldots \mathrm{n})$

The algorithm based on Zaheh is as follows:

$$
\begin{aligned}
& B^{*}=\left(A_{1}^{*} \times A_{2}^{*} \times \ldots \times A_{n}^{*}\right) \circ\left(A_{1}^{*} \times A_{2}^{*} \times \ldots \times A_{n}^{*} \rightarrow B\right) \\
& B^{*}(y)=\underset{\left(x_{1}, \ldots x_{n}\right) \in X_{1} \times X_{2} \ldots \times X n}{\vee}\left[\wedge_{i=1}^{n} A^{*}(x) \wedge\left(\widehat{(}_{i=1}^{n} R_{Z}\left(A_{i}(x), B_{i}(y)\right)\right)\right]
\end{aligned}
$$

The result based on Tsukamoto algorithm is as follows:

$$
\begin{aligned}
& B^{*}=A^{*} \circ\left(A_{i} \rightarrow B\right), \\
& B^{*}(y)=\overbrace{i=1}^{n} \underset{x_{i} \in X_{i}}{\vee}\left[A_{i}^{*}\left(x_{i}\right) \wedge R_{T}\left(A_{i}(x), B_{i}(y)\right)\right]
\end{aligned}
$$

\section{THE BASIC IDEA AND STRUCTURE ANALYSIS OF FUZZY REASONING ALGORITHM}

\section{A. Algorithm thought}

The basic ideas of these algorithms are to transform the reasoning rules described by a group of words into a fuzzy relation, thus realize reasoning algorithm establishment based on fuzzy relation. Analyze the concrete algorithms. No matter what kind of algorithm mode, they all meet the first step of CRI algorithm, which is to transform hypothesis 1 by implication operator. Make it a fuzzy relation $\mathrm{R}(\mathrm{x}, \mathrm{y})$, and then get a solution. Zaheh's basic idea is to take $\mathrm{B} *$ as the basic conclusion of $A *$ and $A \rightarrow B$. This thought is in line with the general logic principles. But on the processing of hypothesis 2, Zadeh made compound operation of hypothesis 1 and 2 with the summarization of CRI algorithms. The process lacks theoretical support, and also affects the original meaning of reasoning. 


\section{B. Algorithm structure}

According to the analysis of algorithm structure, though different implication operators are adopted in different reasoning algorithms, each different fuzzy implication operator is a mapping from $[0,1] \times[0,1]$ to $[0,1]$, and can use fuzzy "or," fuzzy "and", fuzzy "not" to represent the fuzzy relation. Three fuzzy logic relations operators are respectively $t \vee(t-$ complementary module), $t \wedge$ ( $\mathrm{t}$ - mold) and tc (repair), and each fuzzy algorithm's expressions in solving way $\mathrm{B} *(\mathrm{y})$ are basic operators: $\mathrm{t} \vee(\mathrm{t}$ - complementary module), $\mathrm{t} \wedge(\mathrm{t})$ and $\mathrm{tc}$ (repair), so we can get the following conclusion: progress and distance algorithm mind are introduced to multidimensional fuzzy reasoning and multiple fuzzy reasoning algorithm. The results got from rest calculations wtih the classical fuzzy reasoning algorithms are based on the computation results transformation of three operators $\mathrm{t} \vee(\mathrm{t}$ - complementary module), $\mathrm{t} \wedge$ (t-mode) and tc (repair) on $[0,1]$, and the basic algorithm structure is essentially the calculation methods based on the three basic operators. So the calculated results and the objects must have some kind of corresponding mathematical relationship.

\section{THE THEORETICAL BASIS VALIDATION OF FUZZY REASONING ALGORITHM}

For FMP model, A and A * are fuzzy sets on the discourse $\mathrm{X}$. The same, B and B * are the fuzzy sets on $\mathrm{Y}$, and there is a bounded function $\mathrm{F}$ from collection $\mathrm{X}$ to $[0,1]$ for the elements in $\mathrm{X}$ and $\mathrm{Y}$ collection elements: $t \vee(t-$ complementary module), $t \wedge$ (t-mode) and tc (repair). FMP algorithm can get $\mathrm{B} *(\mathrm{Y})$, which is function $\mathrm{F}(\mathrm{X})$.

Certification: Because $\mathrm{A}$ and $\mathrm{A}$ * are the fuzzy sets on $\mathrm{X}$ discourse, the same, $\mathrm{B}$ and $\mathrm{B}$ * are the fuzzy sets on $Y$, then:

$$
A(x), A^{*}(y), B(x), B *(x) \in[0,1], x \in X, y \in Y
$$

Set: $R:[0,1]^{2} \rightarrow[0,1]$

Then hypothesis 1's $A \rightarrow B$ can be converted into $X^{\times} Y^{\prime}$ 's fuzzy relationship based on $R$.

$$
R(x, y)=R(A(x), B(x)),(x, y) \in X \times Y
$$

For FMP, based on three basic operators reasoning, we can get $\mathrm{B} *(\mathrm{y})$, and for hypothesis 1 , there must be a mapping $\mathrm{f}$ from $\mathrm{X} \rightarrow \mathrm{y}$ :

$$
f: X \rightarrow Y, x \mid \rightarrow f(x)=y \in Y
$$

Then the induction is:

$$
f: P(x)^{\rightarrow} P(y) \text { And because } A(x), A^{*}(y), B(x), B^{*}(x) \in[0,1], x \in X, y \in Y
$$

Combining with FMP definition: $A\left|\rightarrow f(A)=B, A^{*}\right| \rightarrow f\left(A^{*}\right)=B^{*}$

So for any elements $\mathrm{X}$ in random $\mathrm{X}$, if $\mathrm{X}$ is the element of $\mathrm{A}{ }^{*}$, there must be a $\mathrm{Y}$ in $\mathrm{Y}$ that $\mathrm{f}(\mathrm{X})$ $=\mathrm{Y} \in \mathrm{B} *$, and because $\mathrm{B} *$ is a fuzzy set on discourse $\mathrm{Y}$, a map can be got:

$u_{B}^{*}: Y^{\rightarrow}[0,1]$, which proves that there is a $u_{B}^{*}$ which meet the demand of the mapping:

$$
\begin{aligned}
& u_{B}^{*} \circ f, \forall_{x} \in Y, \\
& \left(u_{B}^{*} \circ f\right)(x)=u_{B}^{*}(f(x))=u_{B}^{*}(y) \in[0,1]
\end{aligned}
$$


That is:

$\left(u_{B}^{*} \circ f\right)(x)=B^{*}(y)$

Let $\mathrm{F}=\left(u_{B}^{*} \circ f\right)(x)$. Because $A(x), A^{*}(y), B(x), B *(x) \in[0,1], x \in X, y \in Y$, prove that $\mathrm{F}$ is bounded. The prove process and methods are mcFMP and mdFMP are the same. $\mathrm{F}$ (x) can be got by reasoning based on the three basic operators.

\section{Discussion}

Through the reasoning process of multiple fuzzy inference models, the reasoning processes are the calculated results based on $\mathrm{t} \vee(\mathrm{t}$ - complementary module), $\mathrm{t} \wedge$ ( $\mathrm{t}$-mode) and tc (repair) operators, and these "reasoning results" are thought to be bounded function values in [0,1]. The reasoning process carried out at the end of this paper is the proof of the point. And all sorts of fuzzy reasoning algorithms are essentially different structure forms of the bounded function. This paper analyzed fuzzy reasoning algorithm based on the mathematical principle, fully proved that the fuzzy reasoning algorithm has theoretical basis and has a reliable mathematical basis.

\section{REFERENCE}

[1]Ananya Roy,Kaushik Das Sharma. Gravitational Search Algorithm and Lyapunov Theory based Stable Adaptive Fuzzy Logic Controller[J]. Procedia Technology,2013,10:

[2]Kaushik Das Sharma,Amitava Chatterjee,Anjan Rakshit. Harmony search algorithm and Lyapunov theory based hybrid adaptive fuzzy controller for temperature control of air heater system with transport-delay[J]. Applied Soft Computing Journal,2014,25:

[3]Xia Liu,Ling-ge Jiang. A novel vertical handoff algorithm based on fuzzy logic in aid of grey prediction theory in wireless heterogeneous networks[J]. Journal of Shanghai Jiaotong University (Science),2012,171:.

[4] Wang Qingmiao. Fuzzy clustering new algorithm and its application research based on objective function [D]. Jiangsu University, 2014.

[5] Song Hongwei. The design and implementation of super capacitor mixed with battery energy storage system based on fuzzy algorithm [D]. Taiyuan University of Technology, 2014.

[6] Guo Jinsong. Water quality assessment and water quality simulation study Based on artificial neural network (ANN) [D]. Chongqing University, 2002.

[7] Liu Zhanguo. The application research of intelligent control in building air conditioning control system and elevator group control system [D]. Chongqing University, 2008.

[8] Liang Taonian. Fractional order PID controller and parameter uncertainty fractional order system stability domain analysis [D]. Xi 'an University of Electronic Science and Technology, 2011.

[9] Lin Hui. Wheel motor drive electric vehicle combined brake fuzzy self-tuning PID control method research [D]. Jilin University, 2013.

[10] Fu Jinbao. The application research of disturbance observer and advanced control in aerospace camera angle system [D]. Graduate School of Chinese Academy of Sciences (Changchun Optical Precision Machinery and Physical Institute), 2013. 